\title{
Grossesse et risques d'irradiations en radiodiagnostic : état des connaissances des usagers au CHU de Yopougon (Abidjan, Côte d'Ivoire)
}

\author{
N. KOUAMÉ ${ }^{1,2,3 *}$, A.M. NGOAN-DOMOUA ${ }^{1}$, A. SÉTCHÉOU ${ }^{1}$, B.J.P. NEZOU ${ }^{1}$, \\ K.D. KONAN ${ }^{1}$, R.D. N'GBESSO ${ }^{1}$, A.K. KÉITA ${ }^{1}$
}

(Manuscrit reçu le 11 décembre 2011, accepté le 9 mai 2012)

RÉSUMÉ Objectifs : évaluer l'état des connaissances des usagers et des professionnels de la santé sur les risques d'irradiation de la femme enceinte au cours des explorations radiologiques et l'observation de l'application des règles de radioprotection relative à la femme enceinte par les utilisateurs de rayons $\mathrm{X}$. Matériel et méthodes : notre étude prospective, réalisée au CHU de Yopougon, a duré 6 mois. Nous avons interrogé 60 femmes en grossesse ou susceptibles de l'être, 30 médecins prescripteurs non-radiologues, 29 techniciens d'imagerie médicale et 21 aides soignants sur les risques d'irradiation et les mesures de protection de la femme enceinte avant et pendant la réalisation d'un examen radiologique utilisant les rayons $X$. Résultats : $\mathbf{7 3 , 6 8} \%$ des médecins prescripteurs n'ont pas de culture en radioprotection chez une femme enceinte ou en âge de procréer. $93,10 \%$ des patientes enceintes ou en âge de procréer ignoraient l'existence des rayons $\mathrm{X}$ en radiodiagnostic et leur nocivité pour leur état. $80 \%$ des manipulateurs de radiologie ne respectaient pas les règles élémentaires de radioprotection. Conclusion : la sensibilisation des usagers des rayons $X$ sur les risques d'irradiations chez une femme enceinte ou susceptible de l'être est nécessaire au CHU de Yopougon.

ABSTRACT Pregnancy and radiation risks in radiology: users' knowledge at the University Hospital of Yopougon (Abidjan, Côte d'Ivoire).

Objectives: to assess the knowledge of users and health professionals about the risks of irradiation of the pregnant woman in the course of radiological examinations and the application of rules of radiation protection to pregnant women by $\mathrm{X}$-ray users. Materials and Methods: our prospective study, conducted at the University Hospital of Yopougon, lasted 6 months. We interviewed 60 women who were pregnant or likely to be, 30 non-radiologist prescribing physicians, 29 medical imaging technicians and 21 healthcare aides on radiation risk and protective measures for pregnant women before and during the implementation of a radiological examination using X-rays. Results: $73.7 \%$ of prescribing physicians have no knowledge of radiation protection in women who are pregnant or of childbearing age. $93.1 \%$ of patients who are pregnant or of childbearing age were not aware of $\mathrm{X}$-rays in diagnostic radiology and the harm they pose to a pregnant or potentially

\footnotetext{
Service de radiologie CHU de Yopougon, BP 632, Abidjan 21, République de Côte d'Ivoire.

Service de radiologie CHU de Yopougon, BP 2716, Abidjan 21, République de Côte d'Ivoire.

e-mail : kngoran@yahoo.fr

* Auteur correspondant.
} 
pregnant woman. $80 \%$ of radiology manipulators do not respect the basic rules of radiation protection. Conclusion: greater awareness of the users of $\mathrm{X}$-ray radiation risks in pregnancy or women likely to be pregnant is required at the University Hospital of Yopougon.

Keywords: radiation protection / pregnancy / radiation risk / medical radiation exposure

\section{Introduction}

La découverte des rayons X en 1895 par Röntgen a été pour la médecine diagnostique l'une des meilleures, voire la plus grande, avancée de son histoire. Elle a permis d'énormes progrès et continue aujourd'hui avec le scanner à rayons $\mathrm{X}$ son épopée glorieuse tant sur le plan médical que sur les plans sécuritaires et agro-industriels. Mais son utilisation chez l'Homme n'est pas sans risques. En effet, plusieurs études ont montré l'aspect nocif des rayons X notamment dans la genèse des affections malignes et cancéreuses (Smith-Bindman et al., 2009 ; Berrington de González et al., 2009 ; Brenner et Hall, 2007 ; Roudier et al., 2006 ; Cordoliani, 2005) justifiant le terme de « cancer radio-induit ». Ces études soulignent surtout la vulnérabilité du fœtus et donc de la femme enceinte. Selon l'Agence internationale de l'énergie atomique (AIEA) et la Commission internationale de protection radiologique (CIPR), une proportion statistiquement significative des cancers et des leucémies de l'enfance a été constatée après des irradiations au cours de la grossesse (McCollough et al., 2007). Pour cela, la CIPR recommande la mise en place d'un département de radioprotection chargé de veiller au respect des prescriptions et réglementer l'introduction et l'exercice de toute pratique mettant en jeu des sources de rayonnements ionisants, de surveiller les mesures de protection et de sûreté radiologique (ICRP, 1991). Mais la mise en œuvre de ces différentes mesures passe par la formation et l'information des professionnels et des usagers des rayons X, voire de la population générale, sur les risques liés à l'utilisation des rayons X. Aussi avons-nous entrepris cette étude dans l'objectif d'évaluer l'état des connaissances des usagers et des professionnels de la santé sur les risques d'irradiation et l'observation de l'application des règles de radioprotection relative à la femme enceinte à Abidjan (Côte d'Ivoire).

\section{Patients et méthodes}

Notre étude prospective et descriptive a duré 6 mois. Elle a été réalisée du $1^{\mathrm{er}}$ mars 2009 au 31 août 2009 au centre hospitalo-universitaire (CHU) de Yopougon (Abidjan, Côte d'Ivoire). Elle a concerné 60 femmes enceinte ou en âge de procréer, 30 médecins prescripteurs non-radiologues, 29 manipulateurs et 21 aides soignants qui après un consentement éclairé ont accepté de répondre à un questionnaire sur les risques d'irradiation et les mesures de protection de la femme enceinte avant et 
pendant la réalisation d'un examen radiologique utilisant les rayons $\mathrm{X}$ (radiographie standard, urographie intra-veineuse, hystéro-salpingographie, lavement baryté, scanner à rayons X). Le matériel utilisé était constitué de fiches questionnaires et de grilles d'observation relatives aux mesures de radioprotection. L'élaboration du questionnaire et la saisie des données ont été réalisées à l'aide du logiciel sphinx. Le traitement et l'analyse des données ont été effectués sur les logiciels Microsoft world 2007 et Excel 2007.

\section{Résultats}

L'ensemble de nos résultats est détaillé dans les tableaux I-V.

TABLEAU I

Enquête systématique de la femme en âge de procréer sur un éventuel état de grossesse. Systematic examination of women of childbearing age about a possible pregnancy.

\begin{tabular}{lcccccc}
\hline & \multicolumn{2}{c}{ Oui } & \multicolumn{2}{c}{ Non } & \multicolumn{2}{c}{ Total } \\
& Quantité & $\%$ & Quantité & $\%$ & Quantité & $\%$ \\
\hline Médecins prescripteurs & 17 & 56,7 & 13 & 43,3 & 30 & 100 \\
Manipulateurs radio & 5 & 17,2 & 24 & 82,8 & 29 & 100 \\
Aides soignants & 21 & 100 & 0 & 0 & 21 & 100 \\
\hline
\end{tabular}

TABLEAU II

Niveau de connaissance des femmes enceinte ou en âge de procréer sur les rayons $X$ et leurs effets. Level of knowledge of women in pregnancy or of childbearing age on X-rays and their effects.

\begin{tabular}{lcccc}
\hline & Oui & & Non \\
& Quantité & $\%$ & Quantité & $\%$ \\
\hline Avez-vous déjà effectué un examen en radiologie ? & 60 & 100 & 00 & 00 \\
\hline Savez-vous ce que sont les rayons X? & 4 & 6,7 & 56 & 93,3 \\
\hline Selon vous, les rayons X sont-ils nocifs pour le fœtus ? & 13 & 21,7 & 47 & 79,3 \\
\hline Savez-vous qu'une forte dose de rayons X peut être cancérigène ? & 4 & 6,7 & 56 & 93,3 \\
\hline Savez-vous qu'une femme enceinte peut refuser un examen aux rayons X? & 1 & 1,7 & 59 & 98,3 \\
\hline Savez-vous qu'une femme enceinte peut refuser de refaire un examen aux & 1 & 1,7 & 59 & 98,3 \\
rayons X? & & & & \\
\hline Savez-vous qu'il existe des mesures de protection de la femme enceinte ? & 3 & 5 & & 95 \\
\hline Savez-vous qu'une salle de radiologie doit répondre à des normes? & 00 & 00 & 60 & 100 \\
\hline Savez-vous que l'échographie n'est pas dangereuse pour le fœetus ? & 16 & 26,7 & 44 & 73,3 \\
\hline
\end{tabular}


TABLEAU III

Vérification des mesures de radioprotection par les manipulateurs de radiologie. Verification of radiation protection measures by the manipulators of radiology.

\begin{tabular}{lcccc}
\hline & \multicolumn{2}{c}{ Oui } & \multicolumn{2}{c}{ Non } \\
& Quantité & $\%$ & Quantité & $\%$ \\
\hline Vérification de la justification de l'examen & 10 & 34,5 & 19 & 65,5 \\
\hline Utilisation d'un tablier plombé & 12 & 41,4 & 17 & 58,6 \\
\hline Collimation maximale du faisceau de rayons X à la région utile & 10 & 34,5 & 19 & 65,5 \\
\hline Choix de paramètres d'acquisition réduisant la dose au fotus & 10 & 34,5 & 19 & 65,5 \\
\hline Interdiction de scopie sauf nécessité absolue & 25 & 86,2 & 4 & 13,8 \\
\hline Prise de disposition pour éviter des clichés ratés et reprendre l'incidence & 11 & 37,9 & 18 & 62,1 \\
\hline
\end{tabular}

TABLEAU IV

Niveau de connaissance des médecins prescripteurs non-radiologues. Level of knowledge of non-radiologist physicians.

\begin{tabular}{lcccc}
\hline & \multicolumn{2}{c}{ Oui } & \multicolumn{3}{c}{$\begin{array}{c}\text { Non ou absence de } \\
\text { réponse } \\
\end{array}$} & Quantité & $\%$ & Quantité & $\%$ \\
\hline Y a-t-il un risque de cancérogenèse post natal ? & 9 & 30 & 21 & 70 \\
\hline L'irradiation foetale ne justifie quasiment pas d'interrompre la grossesse & 8 & 26,7 & 22 & 73,3 \\
\hline Les effets génétiques des rayons X sont dépendants de la dose-gonade & 5 & 16,7 & 25 & 83,3 \\
\hline $\begin{array}{l}\text { Le gray est l'unité de mesure de la quantité de rayons X absorbée par } \\
\text { l'organisme }\end{array}$ & 6 & 20 & 24 & 80 \\
\hline Y a-t-il des effets biologiques dus aux rayons X? & 9 & 30 & 21 & 70 \\
\hline Le sievert mesure les effets biologiques & 6 & 20 & 24 & 80 \\
\hline Y a-t-il un risque encouru par la mère ? & 3 & 10 & 27 & 90 \\
\hline
\end{tabular}

TABLEAU V

Capacité de substitution des examens utilisant des rayons $\mathbf{X}$ par les médecins prescripteurs. Capacity of substitution of tests using $X$-rays by prescribing physicians.

\begin{tabular}{lcccc}
\hline & \multicolumn{2}{c}{ Oui } & \multicolumn{2}{c}{ Non } \\
& Quantité & $\%$ & Quantité & $\%$ \\
\hline Varices et thromboses chez une gestante & 13 & 43,3 & 17 & 56,7 \\
Lithiase chez une gestante & 14 & 46,7 & 16 & 53,3 \\
Pathologie digestive haute chez une gestante & 14 & 46,7 & 16 & 53,3 \\
Pathologie digestive basse & 11 & 36,7 & 19 & 63,3 \\
\hline
\end{tabular}


Aucun aide-soignant, 82,7 \% des manipulateurs et 42,1\% des médecins prescripteurs non-radiologues n'interrogeaient systématiquement la femme en âge de procréer sur sa date des dernières règles ou sur l'existence éventuelle d'une grossesse.

Toutes les patientes enceintes ou en âge de procréer avaient déjà, au moins une fois, effectué un examen de radiologie. Parmi elles, 93,1 \% n'avaient jamais entendu parler des rayons $\mathrm{X}$ et de leurs effets nocifs sur le fœtus. Aucune des patientes ne savait qu'une salle de radiographie devait répondre à certaines normes et 94,3\% ne connaissaient pas l'existence de mesures de protection pour la femme enceinte en salle d'examen. Seulement 25,9 \% des patientes savaient que l'échographie n'était pas source d'irradiation.

Chez les femmes dont la grossesse était visible, 34,5\% des manipulateurs vérifiaient la justification de l'examen. $41,4 \%$ d'entre eux utilisaient un tablier plombé et 34,5\% déterminaient si le fœus étaient dans le faisceau primaire afin d'utiliser une collimation maximale de faisceau de rayons à la région utile. La même proportion de manipulateurs choisissait des paramètres d'acquisition réduisant la dose au fotus. Aucun manipulateur n'interdisait la scopie. Seulement $37,9 \%$ d'entre eux prenaient des dispositions pour éviter les clichés ratés.

La plupart des médecins prescripteurs de radiologie $(84,2 \%)$ ignoraient l'existence de la Commission internationale de protection radiologique (CIPR). $89,5 \%$ d'entre eux n'avaient pas reçu une formation en radioprotection chez une gestante. $10,5 \%$ des prescripteurs ignoraient l'origine des effets stochastiques et déterministes des rayons X. 57,9\% des prescripteurs ignoraient que le risque de cancérogenèse était fonction de la dose. $47,4 \%$ des prescripteurs ignoraient que l'irradiation fotale résultant des procédures diagnostiques ne justifie pas l'interruption d'une grossesse. 47,4\% des prescripteurs ignoraient l'existence du Gray et du Sievert et ne savaient pas que l'apparition des effets biologiques des rayons X sur l'organisme était fonction de la nature de l'organe exposé. 89,5\% d'entre eux ne savaient pas que le scanner était plus irradiant que la radiologie standard. Sur le plan de la capacité de substitution des examens radiologiques, $57,9 \%$ des médecins prescripteurs ne connaissaient pas de substitut d'examen non-irradiant en cas de varices et de thrombose chez une femme enceinte. En cas de lithiase ou de toute pathologie digestive haute chez une femme enceinte, 52,6\% des médecins prescripteurs ne connaissaient pas de substitut d'examen non irradiant. En cas de pathologie digestive basse, 63,2 \% des médecins prescripteurs ne savaient pas quel examen non irradiant substituer. 


\section{Commentaires}

La prise en charge radiologique de la femme en âge de procréer ou de la femme enceinte ou susceptible de l'être commence depuis la salle de consultation avec le médecin prescripteur jusqu'aux différentes étapes dans le service de radiologie. Dans ce processus, l'enquête systématique sur l'éventuel état de grossesse, ou la recherche de la date des dernières règles, est un élément fondamental pour éviter toute délivrance de rayons X sur l'embryon ou le fœtus ou de prendre certaines précautions pour amoindrir la dose de rayons $\mathrm{X}$ à délivrer. Dans notre étude, près de la moitié des médecins prescripteurs $(42,1 \%)$ ne recherchaient pas cette éventualité. En France, une étude réalisée par Gervaise et al. (2011) a mis en évidence une méconnaissance des mesures de radioprotection chez plus de $30 \%$ des praticiens prescripteurs de scanner. Cette proportion est beaucoup plus élevée chez le personnel de radiologie (100\% des aides soignants et 82,7\% des manipulateurs) à Abidjan (Côte d'Ivoire). Cette insouciance du personnel de radiologie à Abibjan est également observée par le personnel des services de Médecine nucléaire en Australie (James et al., 2011) où l'auteur demande la mise en place de recommandations claires de bonnes pratiques. Selon la littérature (Cordoliani et Foehrenbachi, 2003), la responsabilité de la recherche initiale de la grossesse incombe au médecin demandeur de l'examen et au médecin radiologiste. Pour autant, ce risque pourrait à notre sens avoir plus de chance d'être évité si la femme devant subir l'examen radiologique pouvait préciser au médecin prescripteur et au manipulateur de radiologie, l'existence ou la possibilité d'une éventuelle grossesse. Cette précaution supplémentaire nécessite que cette femme ait l'information sur les rayons $\mathrm{X}$ et leurs effets nocifs sur la grossesse ainsi que l'existence de mesures applicables et effectives de précaution. Dans ce sens, notre étude a montré que 93,1 \% des femmes interrogées ne connaissaient pas l'existence de rayons $\mathrm{X}$ et leurs effets, quoi que toutes aient déjà effectué au moins un examen radiographique dans un passé récent.

Selon le principe ALARA (As Low As Reasonably Achievable), l'utilisation des matériels, des procédures et l'organisation du travail doivent être conçus pour que les expositions soient maintenues aussi bas qu'il est raisonnablement possible et compatible avec la qualité d'image qui apporte l'information médicale recherchée (Gold et Aujard, 2006) ; cela donne une plus large place à l'image utile plutôt qu'à la belle image. Cependant, notre étude a mis en évidence que la plupart des manipulateurs $(65,5 \%)$ observaient des attitudes qui conduisaient à une irradiation incontrôlée du fœetus. La conséquence immédiate est l'engendrement d'une inquiétude disproportionnée et des conduites inadaptées pouvant aboutir à l'extrême : interruption de la grossesse demandée par la patiente ou suggéré à tort par le médecin traitant. Or, selon Elefant et al. (2007), pour des irradiations faibles 
(doses-gonades inférieures à $10 \mathrm{cGy}$ ), les risques de malformations congénitales, d'anomalies fonctionnelles et de cancers induits sont insignifiants. C'est le cas de tous les examens de radiodiagnostic même lorsque l'utérus est dans le faisceau. Malgré cela, nous pensons que, le risque n'étant jamais nul, il est important que des médecins prescripteurs soient formés sur la radioprotection de sorte que les examens radiologiques demandés à la femme enceinte soient utiles. Nous sommes en accord avec McCollough et al. (2007). Selon cet auteur, les effets biologiques potentiels des expositions à des radiations in utero d'un foetus en développement incluent la mort prénatale, le retard de croissance intra-utérin, une microcéphalie, un retard mental, des malformations d'organes, et des cancers infantiles. Le risque de chaque effet dépend de l'âge gestationnel au moment de l'exposition, du fotus, des mécanismes de réparation cellulaire, du niveau d'irradiation et de la dose absorbée. Une comparaison entre les niveaux de dose associée à chacun de ces risques et les doses estimées à partir du foetus des examens radiologiques permettrait de conclure que les risques sont minimes et par conséquent, les examens radiologiques de médecine nucléaire qui peuvent fournir d'importantes informations de diagnostic ne doivent pas être retenu sur les femmes enceintes. Il nous apparaît donc nécessaire, voire indispensable, de substituer un examen radiologique utilisant des rayons $\mathrm{X}$ par des examens tout aussi efficaces voire plus performants mais non irradiants tels que l'échographie couplée au Doppler couleur et l'imagerie par résonance magnétique (IRM) chez la femme enceinte (Wieseler et al., 2010). Dans notre étude, une proportion très importante des médecins prescripteurs interrogés (de 52 à $63,7 \%$ ) ne savaient pas faire cette substitution. Ainsi nous avons entrepris, dans l'immédiat, de mettre en place an sein du service de radiologie une unité de contrôle et de validation des demandes des examens de radiologie et d'informer le personnel médical et paramédical sur l'attitude à avoir concernant l'exécution des examens radiologiques chez les femmes en général et en particulier chez les patientes enceintes ou en âge de procréer.

\section{Conclusion}

Les effets nocifs des radiations ionisantes à usage diagnostique sur le fœtus sont discutés, pourtant la radioprotection des patients et des professionnels de la santé est toujours d'actualité. L'application des mesures de radioprotection prônée par la CIPR et l'AIEA doit être précédée par la formation et l'information de la population et de tous les professionnels de la santé. Elle ne doit pas être uniquement axée sur les médecins radiologues. Notre étude a démontré qu'en Afrique de l'ouest et en particulier à Abidjan, $100 \%$ des femmes enceintes ou susceptibles de l'être et les femmes en âge de procréer ne connaissaient pas l'existence des rayons $\mathrm{X}$ et leurs effets. 57,9\% des médecins prescripteurs d'examens radiographiques ne savaient pas que l'effet des rayons $\mathrm{X}$ sur le fotus 
est fonction de la dose reçue et que $52 \%$ d'entre eux ne savaient pas substituer un examen radiologique irradiant par un autre non irradiant pour une pathologie donnée.

Conflit d'intérêt. Les auteurs déclarent qu'il n'y a aucun conflit d'intérêt.

\section{RÉFÉRENCES}

Berrington de González A., Mahesh M., Kim K.P., Bhargavan M., Lewis R., Mettler F. (2009) Projected cancer risks from computed tomography scans performed in the United States in 2007, Arch. Intern. Med. 69 (22), 2071-2077.

Brenner D.J., Hall E.J. (2007) Computed tomography: an increasing source of radiation exposure, New Engl. J. Med. 357 (22), 2277-2284.

Cordoliani Y.S. (2005) Explorations radiologiques en odontostomatologie. Dosimétrie et estimation du risque, EMC-Stomatologie 1, 163-174.

Cordoliani Y.S., Foehrenbachi H. (2003) Radioprotection, grossesse et exposition médicale, Feuill. Radiol. 43, 154-159.

Elefant E., Cournot M.P., Assari F., Vauzelle C. (2007) Imagerie et grossesse, La lettre du gynécologue 324, 1-9.

Gervaise A., Esperabe-Vignau F., Pernin M., Portron Y., Naulet P., Lapierre-Combes M. (2011) Evaluation des connaissances des prescripteurs de scanner en matière de radioprotection des patients, J. Radiol. 92 (7), 681-687.

Gold F., Aujard Y. (2006) Soins intensifs et réanimation du nouveau-né, Elsevier Masson.

ICRP publication 60 (1991) 1990 Recommendations of International Commission on Radiologic Protection, Ann. ICRP 21 (1-3)

James D.J., Cardew P., Warren-Forward H.M. (2011) Determining the pregnancy status of patients before diagnostic nuclear medecine procedures: the Australian experience, J. Nucl. Med. Technol. 39 (3), 220-225.

McCollough C.H., Schueler B.A., Atwell T.D., Braun N.N., Regner D.M., Brown D.L., LeRoy A.J. (2007) Radiation exposure and pregnancy: when should we be concerned? Radiographics 27 (4), 909-917.

Roudier C., Pirard P., Donadieu J. (2006) Indice des radiodermites secondaires à un geste de radiologie interventionnelle, Presse Méd. 35, 955-959.

Smith-Bindman R., Lipson J., Marcus R., Kim K.P., Mahesh M., Gould R., Berrington de González A., Miglioretti D.L. (2009) Radiation dose associated with common computed tomography exams and the associated lifetime attributed risk of cancer, Arch. Intern. Med. 169 (22), 2078-2086.

Wieseler K.M., Bhargava P., Kanal K.M., Vaidya S., Stewart B.K., Dighe M.K. (2010) Imaging in Pregnant Patients: Examination Appropriateness, Radiographics 30 (5), 1215-1229. 